\title{
Galactic Cosmic Rays from Superbubbles and the Abundances of Lithium, Beryllium, and Boron
}

\author{
Andreu Alibés, Javier Labay, Ramon Canal $^{1}$ \\ Departament d'Astronomia i Meteorologia, Universitat de Barcelona, Martí i Franquès 1, \\ 08028 Barcelona, Spain \\ email: aalibes, javier, ramon@am.ub.es
}

\begin{abstract}
In this article we study the galactic evolution of the LiBeB elements within the framework of a detailed model of the chemical evolution of the Galaxy that includes galactic cosmic ray nucleosynthesis by particles accelerated in superbubbles. The chemical composition of the superbubble consists of varying proportions of ISM and freshly supernova synthesized material. The observational trends of ${ }^{6} \mathrm{LiBeB}$ evolution are nicely reproduced by models in which GCR come from a mixture of $25 \%$ of supernova material with $75 \%$ of ISM, except for ${ }^{6} \mathrm{Li}$, for which maybe an extra source is required at low metallicities. To account for ${ }^{7} \mathrm{Li}$ evolution several additional sources have been considered (neutrino-induced nucleosynthesis, nova outbursts, C-stars). The model fulfills the energetic requirements for GCR acceleration.
\end{abstract}

\section{INTRODUCTION}

The evolution of the light nuclides $\left({ }^{6,7} \mathrm{Li},{ }^{9} \mathrm{Be}\right.$, and $\left.{ }^{10,11} \mathrm{~B}\right)$ has been, since the early 70's, a major topic in the studies of galactic chemical evolution. The theoretical scenario for the synthesis of lithium, beryllium, and boron has remained unchanged for years since the pioneering work of Meneguzzi, Audouze, \& Reeves (1971). Recent observations (see Molaro et al. 1997; Boesgaard et al. 1999b, for instance) have revealed an almost linear correlation of both Be and B with Fe at low metallicities. This new trend seems to rule out the common view of galactic cosmic ray (GCR) particles being accelerated from material of the interstellar medium (ISM), as the latter predicts a quadratic dependence on metallicity, and it has motivated models of LiBeB nucleosynthesis that consider new sites for the production and

\footnotetext{
${ }^{1}$ Institut d'Estudis Espacials de Catalunya, Edifici Nexus, Gran Capità 2-4, 08034 Barcelona, Spain
} 
acceleration of the GCR (Ramaty et al. 2000; Fields et al. 2000; Parizot \& Drury 2000). A source of low-energy cosmic rays, enriched in $\mathrm{C}$ and $\mathrm{O}$, was one of the first suggested solutions to the problem, mainly inspired by gamma-ray observations in Orion which, however, once revised appeared spurious (Bloemen et al. 1999). That has led, temporarily at least, to discard the low-energy component hypothesis.

Scenarios where the composition of the GCR was identical to that of the ejecta from individual supernovae have also been ruled out by observations with the Cosmic Ray Isotope Spectrometer (CRIS) on board of the Advanced Composition Explorer (ACE) that measured the abundance of the radioactive isotope ${ }^{59} \mathrm{Ni}$ and its decay product ${ }^{59} \mathrm{Co}$ in current epoch GCR (Binns et al. 1999). ${ }^{59} \mathrm{Ni}$ decays by electron capture with a half life of $7.6 \times 10^{4}$ years, but it does not decay if it is accelerated. The abundance measurements show a very small amount of ${ }^{59} \mathrm{Ni}$ in GCR, and so there must be a minimum delay of $10^{5}$ years between the explosive nucleosynthesis of the material entering in GCR and its acceleration. Such a delay, however, could be accounted for in models where the GCR originated in a superbubble (SB) region of hot, rarefied, metal-rich gas swept out by the collective effects of massive star winds and supernova explosions.

In the SB scenario of Ramaty et al. (2000) LiBeB nucleosynthesis is a primary mechanism (i.e., production $\propto \mathrm{O}$ ), as the composition of the GCR, that exclusively comes from the ejecta of supernovae inside the SB, does not change in time, and it is quite metal-rich. Other authors preferred a combination of a primary and a secondary (i.e., production $\propto$ $\mathrm{O}^{2}$ ) mechanism. Parizot \& Drury (2000) considered also a SB scenario for the acceleration of GCR, but they adopted a composition of the SB material that is a mixture of ISM plus a 2 or $3 \%$ of SN ejecta, according to non-magnetic SB models. On the other hand, Fields et al. (2000) combined two sources of GCR: one accelerated from the ISM, that has a secondary character, and a second component coming from SB and made of pure ejecta of SNe, having thus a primary behaviour. In the last two cases, the primary mechanism dominates at low metallicities, while the secondary one takes over when the ISM becomes more metal-enriched. Nevertheless, despite that it seemed that the old secondary-only GCR nucleosynthesis paradigm was unable to reproduce the Be and B vs Fe slope, Fields \& Olive (1999) succeeded in doing so by using the recently observed O/Fe relationship, with a single slope $\simeq-0.35$ for $[\mathrm{O} / \mathrm{Fe}]$ vs. $[\mathrm{Fe} / \mathrm{H}]$ at all metallicities (Israelian, García López, \& Rebolo 1998; Boesgaard et al. 1999a; Israelian et al. 2001). The situation about [O/Fe] evolution is, however, far from being clear, as stated in Fulbright \& Kraft (1999).

Most of the models for light element evolution published in recent years used analytical approximations for the evolution of some of the important elements in the LiBeB synthesis: carbon, nitrogen, oxygen, and iron (Fields \& Olive 1999; Fields et al. 2000; Parizot \& Drury 
2000; Ramaty et al. 2000). Some were closed models (Vangioni-Flam et al 1998, Fields \& Olive 1999, Fields et al. 2000), which are unable to solve the G-dwarf problem, as stated in Pagel (1997).

Concerning lithium evolution, several works have recently been published, involving different types of possible stellar sources (Romano et al. 1999), one of which, massive AGB stars, seems to be recently ruled out as an important source (Ventura, D'Antona, \& Mazzitelli 2000). Casuso \& Beckman (2000) have proposed a model that only considers galactic cosmic ray nucleosynthesis, but with a GCR rate proportional to the gas ejection rate from stars with masses lower than $3 \mathrm{M}_{\odot}$.

In this article we analyze the evolution of the light elements by means of a detailed numerical model for the galactic chemical evolution (Alibés, Labay, \& Canal 2001; hereafter ALC). Our model assumes that the Galaxy builds by infall of extragalactic material, in an inside-out scenario for Galaxy formation, and adequately reproduces the main solar neighborhood observational constraints: age-metallicity relation, G-dwarf metallicity distribution, current star formation (SFR) and supernova rates, and the evolution of the main elements up to the iron peak (see figures and tables in ALC). In section 2 the model is briefly described and the results are presented in section 3. The conclusions are given in section 4 .

\section{THE MODEL}

We solve numerically the classical equations for the chemical evolution of the Galaxy, relaxing the instantaneous recycling approximation. The same ingredients as in ALC are used for our LiBeB evolution model. That includes the double infall of Chiappini, Matteucci, \& Gratton (1997) given by:

$$
\frac{d \sigma(t)}{d t}=A e^{-t / \tau_{\mathrm{T}}}+B e^{-\left(t-t_{\max }\right) / \tau_{\mathrm{D}}}
$$

where $\sigma(t)$ is the total surface mass density, $\tau_{\mathrm{T}}$ and $\tau_{\mathrm{D}}$ are the characteristic infall timescales

for the halo-thick disk phase and for the thin disk phase, respectively, and $t_{\max }$ is the time of maximum accretion into the thin disk. We take $t_{\max }=\tau_{\mathrm{T}}=1$ Gyr and $\tau_{\mathrm{D}}=7$ Gyr. The coefficients $A$ and $B$ are fixed by imposing that they reproduce the current solar neighborhood values of the total and halo mass surface densities.

An enriched infall model is adopted, which accretes primordial matter during the first Gyr, and then begins to incorporate slightly enriched material, with a metallicity of $\mathrm{Z}=0.1$ $\mathrm{Z}_{\odot}$, as suggested by the recent observations by Wakker et al. (1999) of a massive $\left(\sim 10^{7} \mathrm{M}_{\odot}\right)$ metal-enriched $\left(0.09 \mathrm{Z}_{\odot}\right)$ cloud that is now falling into the disk. We have also considered the 
more usual assumption of a primordial composition for the whole infall process. Although in the first case there is a small contribution to the light element abundances from the infall, the final results for both types of infall are almost indistinguishable, as it will be shown later.

The initial mass function (IMF), $\Phi$, used is that of Kroupa, Tout, \& Gilmore (1993), which takes different slopes for $\mathrm{M} / \mathrm{M}_{\odot} \leq 0.5,0.5 \leq \mathrm{M} / \mathrm{M}_{\odot} \leq 1.0$, and $\mathrm{M} / \mathrm{M}_{\odot} \geq 1.0$. The range of stellar masses considered goes from 0.08 to $100 \mathrm{M}_{\odot}$.

The star formation rate is from Dopita \& Ryder (1994) (eq. 2) with $m=5 / 3, n=1 / 3$ and $\nu=1.2$ :

$$
\Psi(r, t)=\nu \frac{\sigma^{n}(r, t) \sigma_{\mathrm{g}}^{m}(r, t)}{\sigma^{n+m-1}\left(r_{\odot}, t\right)} \mathrm{M}_{\odot} \mathrm{pc}^{-2} \mathrm{Gyr}^{-1}
$$

applied to the solar galactocentric distance of $8.5 \mathrm{kpc}$.

To account for the CNO evolution some of the most recent and most extensive (both in mass and metallicity) yield calculations are used: van den Hoek \& Groenewegen (1997) for low- and intermediate-mass stars, Woosley \& Weaver $(1995)^{2}$ for type II supernovae and model W7 from Thielemann et al. (1996) for type Ia supernovae. Our results for the CNO evolution are shown in Figure 1 for the two infall compositions. Carbon and nitrogen follow the observational data, while $[\mathrm{O} / \mathrm{Fe}]$ shows a gradual increase towards low metallicity with a slope of $\sim-0.28$. This value is slightly lower than the values obtained from observations of the O I triplet (Abia \& Rebolo 1989; Israelian et al. 1998, 2001; Boesgaard et al. 1999a) of $\approx-0.35$.

\section{1. $\quad$ LiBeB Sources}

In Meneguzzi et al. (1971), only spallation reactions induced by GCR and Big Bang nucleosynthesis of ${ }^{7} \mathrm{Li}$ were considered as sources of LiBeB. Since then, several stellar mechanisms have been proposed as sources of some of the light elements: the $\nu$-process (possible solution to the solar system boron and lithium isotopic ratios problem), nova outbursts (possible producers of ${ }^{7} \mathrm{Li}$ ) and $\mathrm{C}$-stars (identified producers of ${ }^{7} \mathrm{Li}$ ). All of them are included in our evolution code. Massive AGB stars are not included as a source of ${ }^{7} \mathrm{Li}$, since recent works (Ventura et al. 2000) insist on their small contribution to the evolution of this isotope.

\footnotetext{
${ }^{2}$ It is reminded here that, as in our previous solar neighborhood model (ALC), only half the iron yields calculated by these authors is adopted
} 


\subsubsection{Big Bang Nucleosynthesis}

${ }^{7} \mathrm{Li}$ is the only light element that is produced in a significant amount in standard big bang nucleosynthesis (BBN) models. In non-standard BBN, some amount of other isotopes could also be produced, but the data available at low metallicities do not indicate the presence of any kind of abundance plateau for them. A BBN contribution of $\left[{ }^{7} \mathrm{Li}\right]=2.2^{3}$ has been included according to Molaro (1999), which ensures the existence of the lithium or Spite plateau (Spite \& Spite 1982) up to $[\mathrm{Fe} / \mathrm{H}] \approx-1.5$. Due to this BBN production of ${ }^{7} \mathrm{Li}$, open and closed box models of Li evolution do not give the same results, since in closed box models all the primordial lithium is already in place when the evolution begins. Therefore, closed box models with the same nucleosynthetic prescriptions than open ones do produce an overabundance of $\mathrm{Li}$ at $[\mathrm{Fe} / \mathrm{H}]=0$ by a factor $\sim 1.6$. This is not the case for $\mathrm{Be}$ and $\mathrm{B}$, for which open and closed box models give similar results.

\subsubsection{Galactic Cosmic Ray Nucleosynthesis}

As in Parizot \& Drury (2000), we adopt a composition of the Galactic Cosmic Rays $\left(X_{i, \mathrm{GCR}}\right)$ corresponding to their being accelerated inside a superbubble, where newly synthesized material ejected by a supernova $\left(X_{i, \mathrm{ej}}\right)$ is accelerated by the shock waves generated by other SN and mixed with the ISM of that epoch $\left(X_{i, \text { ISM }}\right)$, the evolution of the latter being that shown in Figure 1.

$$
X_{i, \mathrm{GCR}}(t)=\alpha_{\mathrm{ej}} X_{i, \mathrm{ej}}(t)+\left(1-\alpha_{\mathrm{ej}}\right) X_{i, \mathrm{ISM}}(t)
$$

Different compositions of the GCR are used by changing the free parameter $\alpha_{\mathrm{ej}}$ in eq. 3. For $\alpha_{\mathrm{ej}}=0$ we have pure ISM CR composition and for $\alpha_{\mathrm{ej}}=1$, pure SNII ejecta CR composition.

The CR source spectrum considered is the so-called shock acceleration spectrum:

$$
q(E) \propto \frac{p^{-s}}{\beta} e^{-E / E_{0}}
$$

where $p$ and $E$ are the particle momentum and kinetic energy, both per nucleon, $\beta=v / c$ and, for the two parameters, we have chosen $s=2.2$ and $E_{0}=10 \mathrm{GeV} / \mathrm{n}$. $E_{0}$ takes into account the possibility that the spectrum is cut off at high energies. Since a leaky box model

$$
{ }^{3}[\mathrm{X}]=12+\log \left(\frac{X}{H}\right)
$$


for GCR propagation is adopted, a escape path of CR from the Galaxy of $X_{\text {esc }}=10 \mathrm{~g} \mathrm{~cm}^{-2}$ has been included in the propagation calculations.

For the calculation of the production rate by spallation reactions we have used a revised version of the $\mathrm{LiBeB}$ code from Ramaty et al. (1997), which includes the cross sections of Read \& Viola (1984) and takes into account all the possible reactions leading to any $\mathrm{LiBeB}$ isotope, both direct and inverse reactions (including two-step processes like $\left.{ }^{16} \mathrm{O}(p, x){ }^{12} \mathrm{C}\left(p, x^{\prime}\right)^{7} \mathrm{Li}\right)$. Since GCR particles are supposed to come from the material inside a $\mathrm{SB}$, the GCR flux, and therefore the spallation reaction production rate, is made proportional to the Type II supernova rate.

We do not consider any low-energy cosmic rays (LECR), since the only observational evidence of this type of CR, spotted in the Orion star formation region, has been withdrawn (Bloemen et al. 1999). Future X-ray and gamma-ray line observations should clarify whether LECR actually exist.

\subsubsection{The $\nu$-process}

Woosley \& Weaver (1995), in their calculations of the yields from type II supernovae, took into account the contribution from the neutrino-induced nucleosynthesis, i.e. the spallation reactions between the huge flux of neutrinos ejected by the explosion and the material, newly synthesized, in the intermediate layers of the star. This primary mechanism, which has not been observationally corroborated, produces mainly ${ }^{7} \mathrm{Li}$ and ${ }^{11} \mathrm{~B}$, so it could be quite important because GCR nucleosynthesis alone can not reproduce the Solar System lithium and boron isotopic ratios. Since these yields are quite uncertain because the flux intensity and energy spectrum of the neutrinos are not well known, a correction factor, $f_{\nu}$, is introduced, its value being obtained by fitting the meteoritic boron isotopic ratio.

\subsubsection{Novae}

Nova outbursts could be important nucleosynthetic sites for those elements which have overproduction factors there, relative to solar, above 1000. According to the recent yields for CO and ONe nova outbursts from José \& Hernanz (1998), that is the case for ${ }^{7} \mathrm{Li}$ (nova outbursts produce, during the thermonuclear runaway, ${ }^{7} \mathrm{Be}$, which decays into ${ }^{7} \mathrm{Li}$ ). We have, thus, adopted a ${ }^{7} \mathrm{Li}$ average yield per nova outburst of $1.03 \times 10^{-10} \mathrm{M}_{\odot}$, taking into account that $30 \%$ of the nova outbursts come from ONe white dwarfs, and an outburst rate given by the following equation: 


$$
\begin{aligned}
\frac{d R_{\text {outburts }}=}{d t}= & D \int_{M(t)+0.5}^{9.5} \frac{\Phi\left(M_{B}\right)}{M_{B}} d M_{B} \\
& \int_{\mu_{m}}^{\mu_{M}} f(\mu) \Psi\left(t-\tau_{M_{B}(1-\mu)}-t_{\text {cool }}\right) d \mu
\end{aligned}
$$

where $D$ ensures that the current nova outburst rate is $\sim 40 \mathrm{yr}^{-1}$ (Hatano et al. 1997), $M_{B}$ is the binary mass, $\mu$ is the ratio of the mass of the secondary star to that of the whole binary system and finally $f(\mu)$ is the binary mass-ratio distribution function of Greggio \& Renzini (1993). The time for the WD to cool enough to be able to start producing outbursts, $t_{\text {cool }}$, is set to $1 \mathrm{Gyr} .{ }^{7} \mathrm{Li}$ production by nova outbursts could be confirmed by the future INTEGRAL mission.

\subsubsection{C-stars}

Even if supernovae ( $\nu$-process) and novae are theoretically possible sources of ${ }^{7} \mathrm{Li}$, carbon-rich stars (C-stars) with $\mathrm{C} / \mathrm{O}>1$ give the only undeniable evidence for an stellar origin of this isotope, since they are seen to be Li-rich. The majority of them show low carbon isotopic ratios $\left({ }^{12} \mathrm{C} /{ }^{13} \mathrm{C}<15\right)$ and are of $\mathrm{J}$ type (Abia \& Isern 1997), with masses lower that $2-3 \mathrm{M}_{\odot}$. The mechanism by which ${ }^{7} \mathrm{Li}$ is produced and ejected to the ISM is not well established, so the only way to estimate the yield is empirical. Abia, Isern, \& Canal (1993) statistically analyzed the contribution of $\mathrm{C}$-stars to the ${ }^{7} \mathrm{Li}$ nucleosynthesis and suggested a time-dependent production rate:

$$
S_{7}(t)=S_{7} \odot \frac{\int_{M_{\mathrm{l}}}^{M_{\mathrm{u}}} \Phi(M) \Psi\left(t-\tau_{M}\right) d M}{\int_{M_{\mathrm{l}}}^{M_{\mathrm{u}}} \Phi(M) \Psi\left(t_{G}-\tau_{M}\right) d M}
$$

where $M_{\mathrm{l}}=1.2 \mathrm{M}_{\odot}$ and $M_{\mathrm{u}}=3 \mathrm{M}_{\odot}$. They estimated empirically a current production rate, from a sample of galactic C-stars, of $S_{7}^{\odot}=2 \times 10^{-9} \mathrm{M}_{\odot} \mathrm{pc}^{-2} \mathrm{Gyr}^{-1}$. The authors point out that this ${ }^{7} \mathrm{Li}$ production rate can only be a lower limit and they needed to increase it to $6 \times 10^{-8} \mathrm{M}_{\odot} \mathrm{pc}^{-2} \mathrm{Gyr}^{-1}$ (Abia, Isern \& Canal 1995) in order to obtain a good fit to the solar system data. In the present model, this rate has only to be increased up to $1.5 \times 10^{-8}$ $\mathrm{M}_{\odot} \mathrm{pc}^{-2} \mathrm{Gyr}^{-1}$. 


\subsection{Destruction of $\mathrm{LiBeB}$}

Traditionally, LiBeB isotopes are supposed to be completely destroyed in stars, so when they die they do not contribute to the ISM abundance. This assumption has been checked by means of main-sequence stellar models (Hansen \& Kawaler 1994) that include all the nuclear reactions involved in $\mathrm{LiBeB}$ destruction, whose rates are taken from Caughlan \& Fowler (1988). We have evaluated, for the whole range of stellar masses, the fraction of each LiBeB isotope that survives and is ejected from the star. Our results are displayed in Figure 2. As expected, the two lithium isotopes are the most fragile ones within the LiBeB group, and they are almost completely destroyed in stars over a wide range of stellar masses. Beryllium and both boron isotopes are more resistant, since they are destroyed at higher temperatures, but nevertheless the fraction of the original BeB that survives is, as in the case of the lithium isotopes, very small.

These destruction terms are included in our calculations, and there are only minute differences in the final results as compared with those obtained assuming complete destruction.

\section{RESULTS}

We have analyzed the evolution of the light elements, taking into account all the sources described in section 2.1 and the restrictions that energetics puts on spectrum and composition of GCR. In Table 1 a summary of all the different sources considered is presented. It is important to notice that no other combination of the contribution from the different sources could reproduce all the constraints considered (Li plateau, beryllium evolution versus iron, and solar system boron isotopic ratio and lithium abundance).

\section{Table 1: SUMMARY OF THE DIFFERENT SOURCES CONSIDERED}

\begin{tabular}{lcll}
\hline \hline source & isotopes produced & contribution & fixed by \\
\hline BBN & ${ }^{7} \mathrm{Li}$ & {$\left[{ }^{7} \mathrm{Li}\right]=2.2$} & $\mathrm{Li}$ plateau \\
GCRN & $\mathrm{LiBeB}$ & $\alpha_{\mathrm{ej}}=0.25$ & Be vs Fe \\
$\nu$-process & ${ }^{7} \mathrm{Li}$ and ${ }^{11} \mathrm{~B}$ & $f_{\nu}=0.29$ & $\left({ }^{11} \mathrm{~B} /{ }^{10} \mathrm{~B}\right)_{\odot}$ \\
novae & ${ }^{7} \mathrm{Li}$ & averaged yield & - \\
C-stars & ${ }^{7} \mathrm{Li}$ & $S_{7}^{\odot}=1.5 \times 10^{-8} \mathrm{M}_{\odot} \mathrm{pc}^{-2} \mathrm{Gyr}^{-1}$ & $\mathrm{Li}_{\odot}$ \\
\hline
\end{tabular}




\subsection{Abundances Evolution}

Concerning the GCR composition, we show in the left panel of Figure 3 that for values of $\alpha_{\mathrm{ej}}$ (see eq. 3) near 0.25 (solid line) good fits to the observed Be and B evolution as a function of $[\mathrm{Fe} / \mathrm{H}]$ are obtained. The $\chi^{2}$ test gives an interval for the possible $\alpha_{\mathrm{ej}}$ values of $(0.12,0.41)$ for a level of confidence of $90 \%$. For values of $\alpha_{\mathrm{ej}}$ near 0 (GCR made only from accelerated ISM), a slope of 2 results, while, if taking a value of $\alpha_{\text {ej }}$ close to 1 (GCR made of SNe ejecta) the contribution from GCR nucleosynthesis to LiBeB evolution at low metallicities is too large. The preceding means that a double origin for the composition of GCR must be considered, since both a pure SNII composition and a pure ISM composition can be rejected. Such a mixture is not well explained in non-magnetic SB models (which favour $\alpha_{\text {ej }} \approx 0.02$ ), unless we assume that the bulk of the GCR comes from the central part of the SB, where the SN ejecta material would be more dominant (Higdon et al. 1998). However, magnetic SB models (Tomisaka 1992) give higher values for $\alpha_{\mathrm{ej}}$, because magnetic fields reduce heat conduction, and thus the evaporation of the ISM shell surrounding the SB. The value of $\alpha_{\mathrm{ej}}$ for these latter models is close to our best fit. In the right panel of Figure 3 , we compare the evolution, in the $\alpha_{\mathrm{ej}}=0.25$ case, for the two different infall compositions. One sees that the evolution is practically unaffected by the chemical composition of the accreted matter, as long as its enrichment remains moderate.

The very recent beryllium abundance determination in the very metal-poor halo star $\mathrm{G}$ $64-12([\mathrm{Fe} / \mathrm{H}]=-3.30$ and $[\mathrm{Be}]=-1.1)$ by Primas et al. $(2000)$ is not well fitted. As stated by the authors, this value would either suggest a flattening of the Be vs Fe relationship at low metallicities or dispersion of values in the early Galaxy. More low-metallicity data are needed to confirm either of these suggestions.

Parizot (2000), also within the framework of a SB model for the acceleration of GCR, analyzed the proportion of pure ejecta of supernovae accelerated in superbubbles (our parameter $\left.\alpha_{\mathrm{ej}}\right)$. He found a best value of 0.03 when adopting a GCR energy spectrum $q(E) \propto E^{-1} \exp \left(-E / E_{0}\right)$ with a rather low cut-off energy of $E_{0}=500 \mathrm{MeV} / \mathrm{n}$, that is supposed to mimic the results of particle acceleration in SB by a collection of weak shocks. Thus, our best value of $\alpha_{\mathrm{ej}}=0.25$ is a factor of $\sim 8$ larger than the result obtained by Parizot (2000). Such discrepancy comes in part from the higher efficiency of his weak SB spectrum. Had this spectrum been used instead of that in eq. (4), a larger Be production per erg would have been obtained, by a factor of $\sim 2.2$, thus reducing by the same factor our mixing coefficient $\alpha_{\mathrm{ej}}$. A further factor of $\sim 3$ is due to the fact that Parizot (2000) did not calculate the evolution of the actual $\mathrm{Be} / \mathrm{O}$ abundance ratio, but only that of the $\mathrm{Be} / \mathrm{O}$ production

ratio by dividing the yields of $\mathrm{Be}$ and $\mathrm{O}$ per supernova. In that way, stellar astration, which is important for late times and high metallicities, was not taken into account. Astration 
severely reduces the abundance of $\mathrm{Be}$, but not that of $\mathrm{O}$, thus lowering the $\mathrm{Be} / \mathrm{O}$ abundance ratio at late times (Fields et al. 2001). Our model, neglecting stellar astration, produces a Be abundance at $[\mathrm{Fe} / \mathrm{H}]=0$ which is $\sim 3$ times larger than the value measured in the Solar System. Finally, the study by Parizot (2000) does not follow the detailed evolution of the composition of the ISM by means of a chemical evolution model. Instead, he assumes solar composition scaled with metallicity, i.e. $\mathrm{O} / \mathrm{H}$, which translates into overestimated $\mathrm{C}$ and $\mathrm{N}$ abundances, both in the ISM and in the $1-\alpha_{\mathrm{ej}}$ fraction of GCR accelerated from the ISM.

Since beryllium is supposed to be produced only by GCR, and therefore its abundance is expected to be directly dependent on the oxygen abundance in the ISM, it is interesting to compare our model results with the observational data in a $[\mathrm{Be}]$ vs $[\mathrm{O} / \mathrm{H}]$ plot (Fig. 4). We notice that if we use the oxygen calculated in ALC, the best value of the parameter $\alpha_{\text {ej }}$ does not seem to be the same as that obtained from the $[\mathrm{Be}]$ vs $[\mathrm{Fe} / \mathrm{H}]$ plot. This flaw was expected since our calculated oxygen evolution does not perfectly fit the Israelian et al. $(1998,2001)$ and the Boesgaard et al. (1999a) data, although our results are closer to them than those obtained in other recently published galactic chemical evolution models. However, when their relationship for $[\mathrm{O} / \mathrm{Fe}]$ vs $[\mathrm{Fe} / \mathrm{H}]$ is used to transform the Be-Fe plot to a Be-O one, a reasonable fit is recovered for $\alpha_{\mathrm{ej}}=0.25$, at least for the low metallicity region where our results closely follow the mean trend of the data. At high metallicity we slightly overpredict the Boesgaard et al. (1999b) data, but the Solar System value is well reproduced still.

In Figure 5, the evolution of total lithium and ${ }^{6} \mathrm{Li}$ are shown: in the left panel for several values of the parameter $\alpha_{\mathrm{ej}}$ and in the right panel for the two infall compositions and fixed $\alpha_{\mathrm{ej}}$. The lithium plateau is well reproduced, as well as the upper envelope of the population I stars and the Solar System value. As shown in Figure 6, the increase of the lithium abundance for $[\mathrm{Fe} / \mathrm{H}]>-1.5$ is due to the contribution from the neutrino-induced nucleosynthesis, and continues by the additional Li from $\mathrm{C}$-stars and nova outbursts, whose contribution is delayed to intermediate and high metallicities due to the long lifetimes of the low-mass progenitors of those objects. C-stars are the main contributors at high metallicities, as it is clearly seen in the Figure. The parameter $\alpha_{\mathrm{ej}}$ has little effect on the lithium evolution, because at low metallicities, where changes of this parameter make the GCR nucleosynthesis production rate change, the Li from the Big Bang and from the $\alpha+\alpha$ reactions dominate. In the case of ${ }^{6} \mathrm{Li}$, the less abundant lithium isotope and still not sufficiently measured at low metallicities, our model reproduces the Solar System value, but not the low-metallicity observations. As indicated by Ramaty et al. (2000), this may suggest the need for an extra source of ${ }^{6} \mathrm{Li}$ in the Galaxy. A possible solution to this defect of ${ }^{6} \mathrm{Li}$ production would be a higher primordial value (some non-standard BBN models produce large ${ }^{6} \mathrm{Li}$ abundances: Jedamzik \& Rehm 2001), but that would mean the existence of plateaus at low metallicities 
also for Be and B (or a ${ }^{7} \mathrm{Li}$ primordial value not compatible with the Spite plateau), which have not been observed up to now.

As it happens for $\mathrm{Be}$ and $\mathrm{B}$, the $\mathrm{Li}$ evolution is almost independent of the assumed composition of the infalling matter.

\subsection{Ratios Evolution}

Isotopic and elemental ratios are crucial to analyze the importance of the different contributions to $\mathrm{LiBeB}$ abundances. The boron isotopic ratio shows, for a given value of $\alpha_{\mathrm{ej}}$, the importance of the neutrino induced nucleosynthesis. This source is necessary to reach the solar system value. On the other hand, the boron over beryllium ratio data are quite constant at all metallicities, implying that the $\nu$-process can not be dominant. The data on the next element ratio considered, $\mathrm{Li} / \mathrm{B}$, show a steep descent from the large value at low metallicities, due to the Li plateau, down to the solar value. Finally, there are quite few available data for ${ }^{6} \mathrm{Li}$, so the lithium isotopic data are not a strong constraint, the solar system data excepted.

In all cases we have used a value of the parameter that restricts the contribution of the neutrino induced nucleosynthesis of $f_{\nu}=0.29$ (similar to that found by Ramaty et al. 2000), which allows to reproduce the Solar System boron isotopic ratio, as displayed in the upper panel of Figure 7 . Higher values of $f_{\nu}$ would increase the ratio ${ }^{11} \mathrm{~B} /{ }^{10} \mathrm{~B}$ up to values not allowed by the Solar System data. We see that the ratio is quite constant during the halo phase (a higher ratio for lower values of $\alpha_{\mathrm{ej}}$ ) due to the contribution of the $\nu$-process and the primary character of GCR nucleosynthesis in those early epochs, and it decreases towards the Solar System value when the secondary behaviour of the GCR becomes important enough. The lack of data at low metallicity hinders a more firm conclusion, but the descending trend after $[\mathrm{Fe} / \mathrm{H}]=0$ is compatible with the current ISM ratio (3.4 \pm 0.7 , Lambert et al. 1998).

A similar behavior is shown by the $\mathrm{B} /$ Be ratio (lower panel of Fig. 7). The constant ratio is higher for lower values of $\alpha_{\mathrm{ej}}$, and the standard GCR nucleosynthesis also dominates at high metallicity.

Finally, the two other ratios considered include lithium (Figure 8 , with ${ }^{6} \mathrm{Li} /{ }^{7} \mathrm{Li}$ in the upper panel and $\mathrm{Li} / \mathrm{B}$ in the lower one). As we have already pointed out, our model can not account for the ${ }^{6} \mathrm{Li}$ at low metallicities, so the lithium isotopic ratio obtained is much lower than the nonzero ${ }^{6} \mathrm{Li} /{ }^{7} \mathrm{Li}$ data around $[\mathrm{Fe} / \mathrm{H}]=-2.5$. The values at intermediate metallicities are not reproduced, since the stars where they have been measured may have suffered some amount of lithium destruction (more important in the case of ${ }^{6} \mathrm{Li}$ than for ${ }^{7} \mathrm{Li}$ ). 
For the $\mathrm{Li} / \mathrm{B}$ ratio this lack of ${ }^{6} \mathrm{Li}$ is not important and our models fit well the available data.

\subsection{Energetics}

Since supernovae, either individually or in SB, are supposed to be the site of GCR acceleration, the total energy of a SN explosion must be larger than the energy of the GCR particles produced by it. The mean value of the Be/Fe ratio in low-metallicity stars measured by Molaro et al. (1997) and Boesgaard et al. (1999b) is about $1.37 \times 10^{-6}$ and the average yield of ${ }^{56} \mathrm{Fe}$ from $\mathrm{SN}$, according to ALC prescriptions, is $Q_{\mathrm{SN}}(\mathrm{Fe})=0.0515 \mathrm{M}_{\odot} \equiv 1.1 \times 10^{54}$ atoms. Then, as shown by Ramaty et al. (1997), one can evaluate the number of atoms of Be produced by each SN event:

$$
Q_{\mathrm{SN}}(\mathrm{Be})=\frac{\mathrm{Be}}{\mathrm{Fe}} Q_{\mathrm{SN}}(\mathrm{Fe}) \simeq 1.5 \times 10^{48} \text { atoms }
$$

We have also evaluated the number of atoms of beryllium produced per erg $(\dot{Q}(\mathrm{Be}) / \dot{W}$, upper panel in Figure 9), so we can show in the lower panel the amount of energy per SN required to accelerate $\mathrm{GCR}$, as

$$
W_{\mathrm{GCR}}^{\mathrm{SN}}=\frac{Q_{\mathrm{SN}}(\mathrm{Be})}{\dot{Q}(\mathrm{Be}) / \dot{W}}
$$

Since the early Galaxy was poor on metals, more energy per SN was required to account for the $\mathrm{Be} / \mathrm{Fe}$ ratio than in the present day, when the secondary mechanism also contribute to the synthesis of Be. In the halo phase, the value of $W_{\mathrm{GCR}}^{\mathrm{SN}}$ is about $2 \times 10^{50} \mathrm{erg}$, so the energy constraint is fulfilled because only 15-20\% of the kinetic energy of any supernova has to be involved in GCR acceleration.

\section{CONCLUSIONS}

The results of a recent and successful galactic chemical evolution model are applied to the particular problem of the light elements. To calculate the LiBeB evolution, it is necessary to know which are the abundances of hydrogen, helium, and the CNO nuclei in the ISM, since they are the main targets of the GCR to produce lithium, beryllium, and boron. The evolution of iron is also important, because traditionally the light element evolution has been plotted in $[\mathrm{LiBeB}]$ vs $[\mathrm{Fe} / \mathrm{H}]$ graphs and iron has been taken as representative of the

metallicity of the stars where LiBeB have been measured. In order to achieve this goal, we 
have used some of the latest and most complete calculations of the yields from different types of stellar scenarios for nucleosynthesis: low- and intermediate-mass stars, Type Ia and II supernovae, and nova outbursts.

Our calculations show that a mixture of $25 \%$ of SN ejecta (with upper and lower limits of $12 \%$ and $41 \%$, respectively) and $75 \%$ of ISM, for the GCR composition, can account for the linear trend of $\mathrm{BeB}$ vs Fe. If the $\mathrm{O} / \mathrm{Fe}$ vs Fe relationship of Boesgaard et al. (1999a) (somewhat steeper than the one obtained by ALC) is used, an acceptable fit to the Be vs $\mathrm{O}$ plot is also obtained.

On the other side, the lithium plateau is easily reproduced with a BBN contribution, as usual, and the increase of the Li abundance after the halo phase is quite nicely reproduced as well by taking into account several sources (the $\nu$-process, nova outbursts, and C-stars).

Concerning the isotopic evolution, our model fails to reproduce the early-time ${ }^{6} \mathrm{Li} /{ }^{7} \mathrm{Li}$ ratio, due to a lack of ${ }^{6} \mathrm{Li}$ at low metallicities. We point out the necessity of an extra source for this isotope. The other calculated ratios, however, nicely reproduce the data.

Finally, the energy problem has been addressed, in order to check that not too large a fraction of the supernova energy is required to produce the light elements.

This work has been supported by the DGESIC grant PB98-1183-C03-01 and the DGI

grant AYA2000-0983. We thank late Dr. R. Ramaty for kindly providing us with his numerical code for cosmic-ray induced nucleosynthesis of the light elements.

\section{REFERENCES}

Abia, C., \& Rebolo, R., 1989, ApJ, 347, 186

Abia, C., Isern, J., \& Canal, R. 1993, A\&A, 275, 96

Abia, C., Isern, J., \& Canal, R. 1995, A\&A, 298, 465

Abia, C., \& Isern, J. 1997, MNRAS, 289, L11

Alibés, A., Labay, J., \& Canal, R. 2001, A\&A, 370, 1103 (ALC)

Barbuy, B., \& Erdelyi-Mendes, M. 1989, A\&A, 214, 239

Binns, W.R., et al. 1999, in LiBeB, Cosmic Rays and Related X- and Gamma-rays, ed. R. Ramaty, E. Vangioni-Flam, M. Cassé, and K. Olive, 96

Bloemen, H. et al. 1999, ApJ, 521, L137

Bonifacio, P., \& Molaro, P. 1997, MNRAS, 285, 847 
Boesgaard, A.M., King, J.R., Deliyannis, C.P., \& Vogt, S.S. 1999a, AJ, 117, 492

Boesgaard, A.M., Deliyannis, C.P., King, J.R., Ryan, S.G., Vogt, S.S., \& Beers, T.C. 1999b, AJ, 117, 1549

Carbon, D.F., Barbuy, B., Kraft, R.P., Friel, E.D., \& Suntzeff, N.B. 1987, PASP, 99, 335

Carretta, E., Gratton, R.G., \& Sneden, C. 2000, A\&A, 356, 238

Casuso, E., \& Beckman, J.E. 2000, PASP, 112, 942

Caughlan, G.R., \& Fowler, W.A. 1988, At. Data Nucl. Data Tables, 40, 284

Chen, Y.Q., Nissen, P.E., Zhao, G., Zhang, H.W., \& Benomi, T. 2000, A\&AS, 141, 491

Chen, Y.Q., Nissen, P.E., Benoni, T., \& Zhao, G. 2001, A\&A, in press

Chiappini, C., Matteucci, F., \& Gratton, G. 1997, ApJ, 477, 765

Cunha, K., Smith, V.V., Boesgaard, A.M., \& Lambert, D.L. 2000, ApJ, 530, 939

Dopita, M.A., \& Ryder, S.D. 1994, ApJ, 430, 163

Edvardsson, B., Anderson, J., Gustafsson, B., Lambert, D.L., Nissen, P.E., \& Tomkin, J. 1993, A\&A, 275, 101

Fields, B.D., \& Olive, K.A. 1999, ApJ, 516, 797

Fields, B.D., Olive, K.A., Vangioni-Flam, E., \& Cassé, M. 2000, ApJ, 540, 930

Fields, B.D., Olive, K.A., Cassé, M., \& Vangioni-Flam, E. 2001, A\&A, 370, 623

Fulbright, J.P., \& Kraft, R.P. 1999, AJ, 118, 527

Fulbright, J.P. 2000, AJ, 120, 1841

García López, R.J., Lambert, D.L., Edvardsson, B., Gustafsson, B., Kiselman, D., \& Rebolo, R. 1998, ApJ, 500, 241

Gratton, R.G. 1985, A\&A, 148, 105

Gratton, R.G., \& Ortolani, S. 1986, A\&A, 169, 201

Greggio, L., \& Renzini, A. 1983, A\&A, 118, 217

Grevesse, N., \& Sauval, A.J. 1998, Space Science Reviews, 85, 161

Hansen, C.J., \& Kawaler, S.D. 1994, Stellar Interiors. Physical Principles, Structure, and Evolution, Springer-Verlag, New York

Hatano, K., Branch, D., Fisher, A., \& Starfield, S. 1997, MNRAS, 290, 113

Higdon, J.C., Lingenfelter, R.E, \& Ramaty, R. 1998, ApJ509, 33

Hobbs, L.M., \& Thorburn, J.A. 1997, ApJ, 491, 772 
Hobbs, L.M. 1999, in LiBeB, Cosmic Rays and Related X- and Gamma-rays, ed. R. Ramaty, E. Vangioni-Flam, M. Cassé, \& K. Olive, 23

Israelian, G., García López, R., \& Rebolo, R. 1998, ApJ, 507, 805

Israelian, G., Rebolo, R., García López, R., Bonifacio, P., Molaro, P., Basri, G., \& Shchukina, N. 2001, ApJ, 551, 833

Jedamzik, K., \& Rehm, J.B. 2001, Phys. Rev. D, 64, 023510

José, J., \& Hernanz, M. 1998, ApJ, 494, 680

Kroupa, P., Tout, C., \& Gilmore, G. 1993, MNRAS, 262, 545

Laird, J.B. 1985, ApJ, 289, 556

Lambert, D.L., Sheffer, Y., Federman, S.R., Cardelli, J.A., Sofia, U.J., \& Knauth, D.C. 1998, ApJ, 494, 614

McWilliam, A., Preston, G.W., Sneden, C., \& Searle, L. 1995, AJ, 109, 2757

Meneguzzi, M., Audouze, J., \& Reeves, H. 1971, A\&A, 15, 337

Mishenina, T.V., Korotin, S.A., Klochkova, V.G., \& Panchuk, V.E. 2000, A\&A, 353, 978

Molaro, P., Bonifacio, P., Castelli, F., \& Pasquini, L. 1997, A\&A, 219, 593

Molaro, P. 1999, in LiBeB, Cosmic Rays and Related X- and Gamma-rays, ed. R. Ramaty, E. Vangioni-Flam, M. Cassé, \& K. Olive , 6

Nissen, P.E., Gustafsson, B., Edvardsson, B., \& Gilmore, G. 1994, A\&A, 285, 440

Nissen, P.E., Lambert, D.L., Primas, F., \& Smith, V.V. 1999, A\&A, 348, 211

Nissen, P.E., Chen, Y.Q., Schuster, W.J., \& Zhao, G. 2000, A\&A, 353, 722

Pagel, B. 1997, Nucleosynthesis and Galactic Chemical Evolution, Cambridge University Press

Parizot, E. 2000, A\&A, 362, 786

Parizot, E., \& Drury, L. 2000, in Proc. IAU Symp. 198, The Light Elements and Their Evolution, ed. L. da Silva, M. Spite, and J.R. de Medeiros, 35

Peterson, R.C., Kurucz, R.L., \& Carney, B.W. 1990, ApJ, 350, 173

Primas, F., Duncan, D.K., Peterson, R.C., \& Thorburn, J.A. 1999, A\&A, 343, 545

Primas, F., Asplund, M., Nissen, P.E., \& Hill, V. 2000 A\&A364, L42

Ramaty, R., Kozlovsky, B., Lingenfelter, R.E., \& Reeves, H. 1997, ApJ, 488, 730

Ramaty, R., Scully, S.T., Lingenfelter, R.E., \& Kozlovsky, B. 2000, ApJ, 534, 747

Read, S., \& Viola, V. 1984, Atomic Data and Nucl. Data Tables, 31, 359 
Romano, D., Matteucci, F., Molaro, P., \& Bonifacio, P. 1999, A\&A, 352, 117

Ryan, S.G., Norris, J.E., \& Beers, T.C. 1999, ApJ, 523, 654

Ryan, S.G., Kajino, T., Beers, T.C., Suzuki, T.K., Romano, D., Matteucci, F., \& Rosolankova, K. 2001 ApJ, 549, 55

Smith, V.V., Lambert, D.L., \& Nissen, P.E. 1998, ApJ, 506, 405

Spite, F., \& Spite, M. 1982, A\&A, 115, 357

Thielemann, F.-K., Nomoto, K., \& Hashimoto, M. 1996, ApJ, 460, 408

Tomisaka, K. 1992, PASJ, 44, 177

Tomkin, J., Sneden, C., \& Lambert, D.L. 1986, ApJ, 302, 415

van den Hoek, L.B., \& Groenewegen, M.A.T. 1997, A\&AS, 123, 305

Vangioni-Flam, E., Ramaty, R., Olive, K.A., \& Cassé, M. 1998, A\&A, 337, 714

Ventura, P., D’Antona, F., \& Mazzitelli, I. 2000, A\&A, 363, 605

Wakker, B.P. et al. 1999, Nature, 402, 388

Woosley, S.E., \& Weaver, T.A. 1995, ApJS, 101, 181 


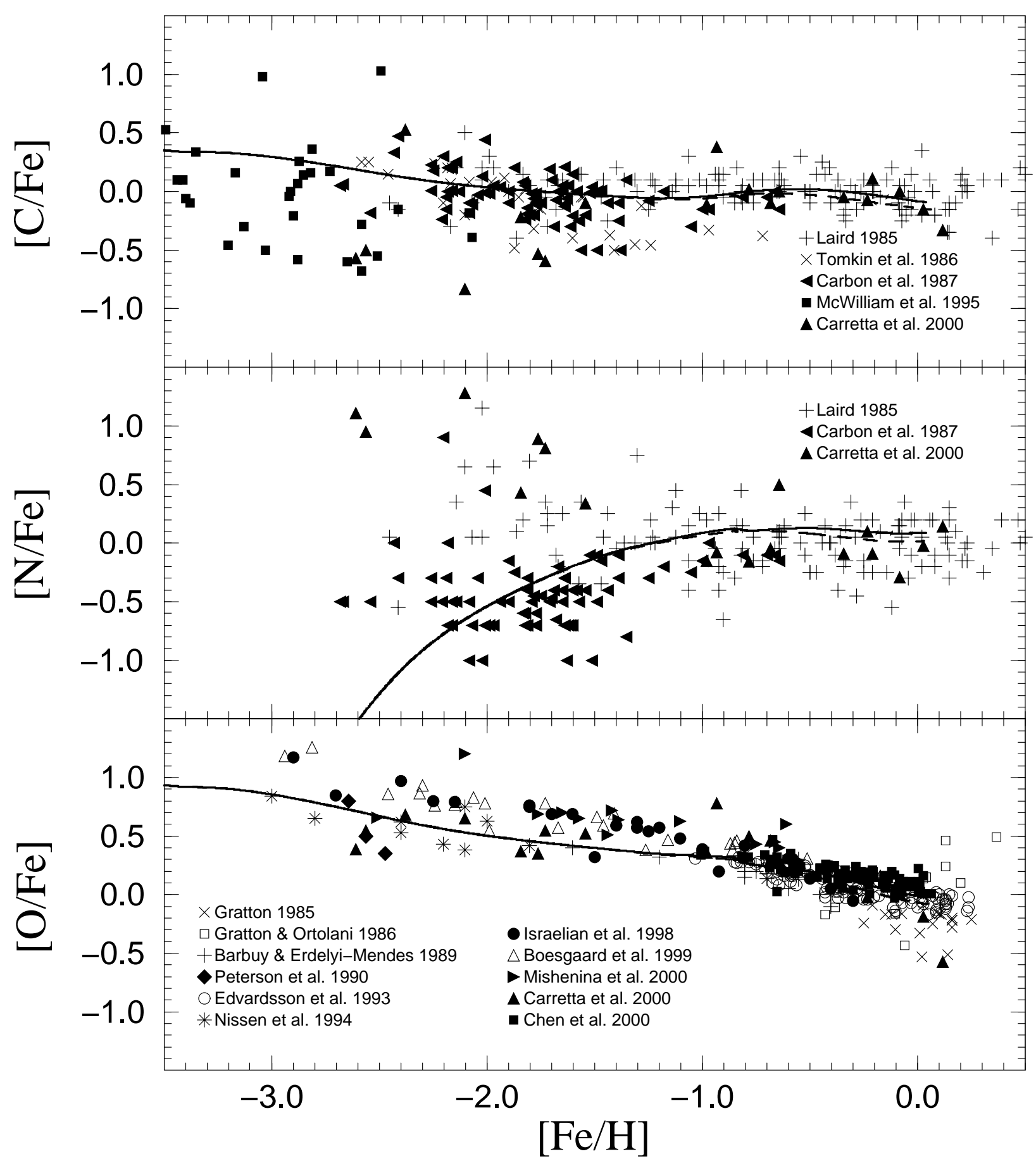

Fig. 1.- Carbon, nitrogen, and oxygen evolution, from ALC. Solid line: enriched model; dashed line: primordial model. 


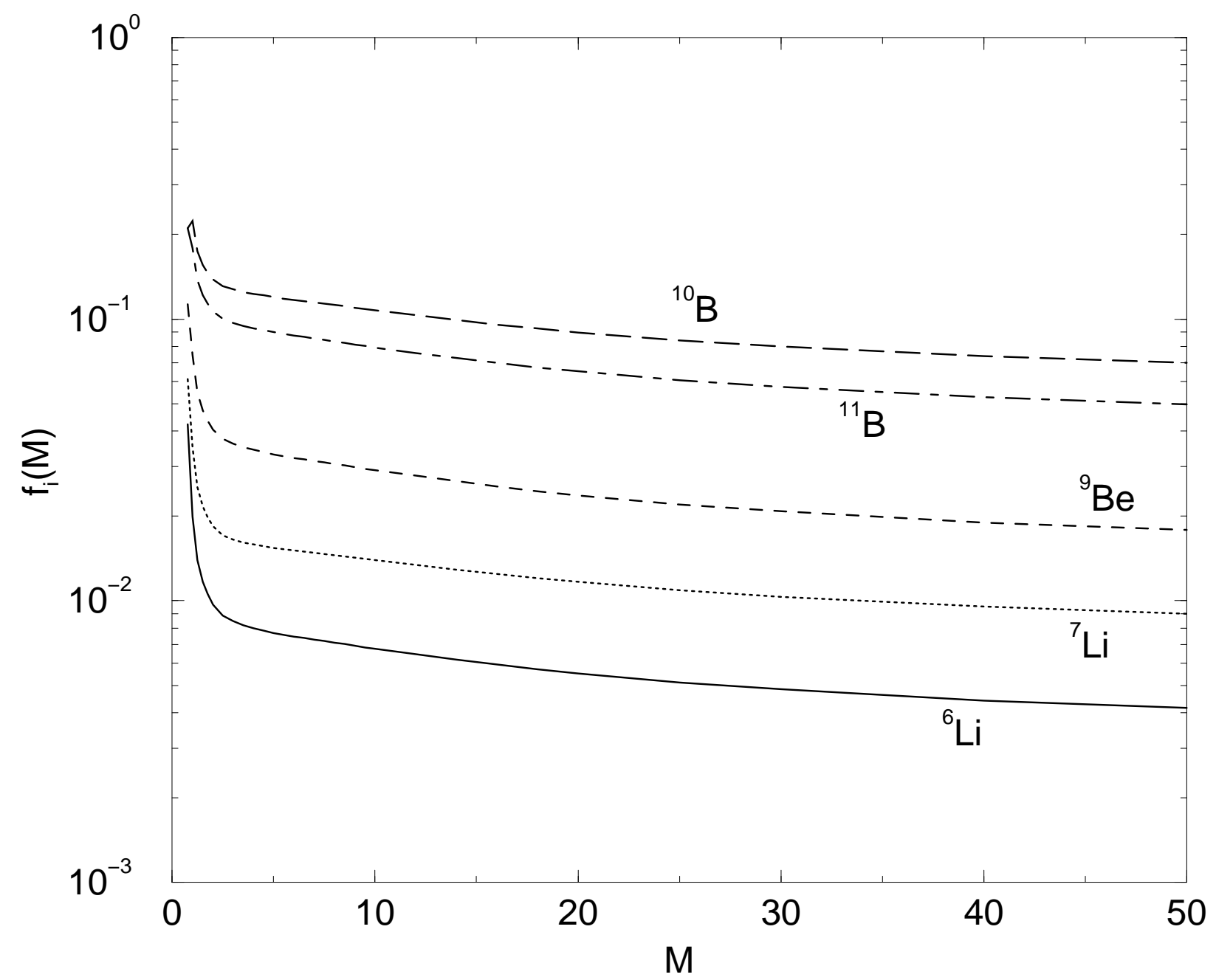

Fig. 2.- Astration model results. Mass fraction of a LiBeB isotope that has survived and has been ejected from each star $\left(f_{i}(M)\right)$. 

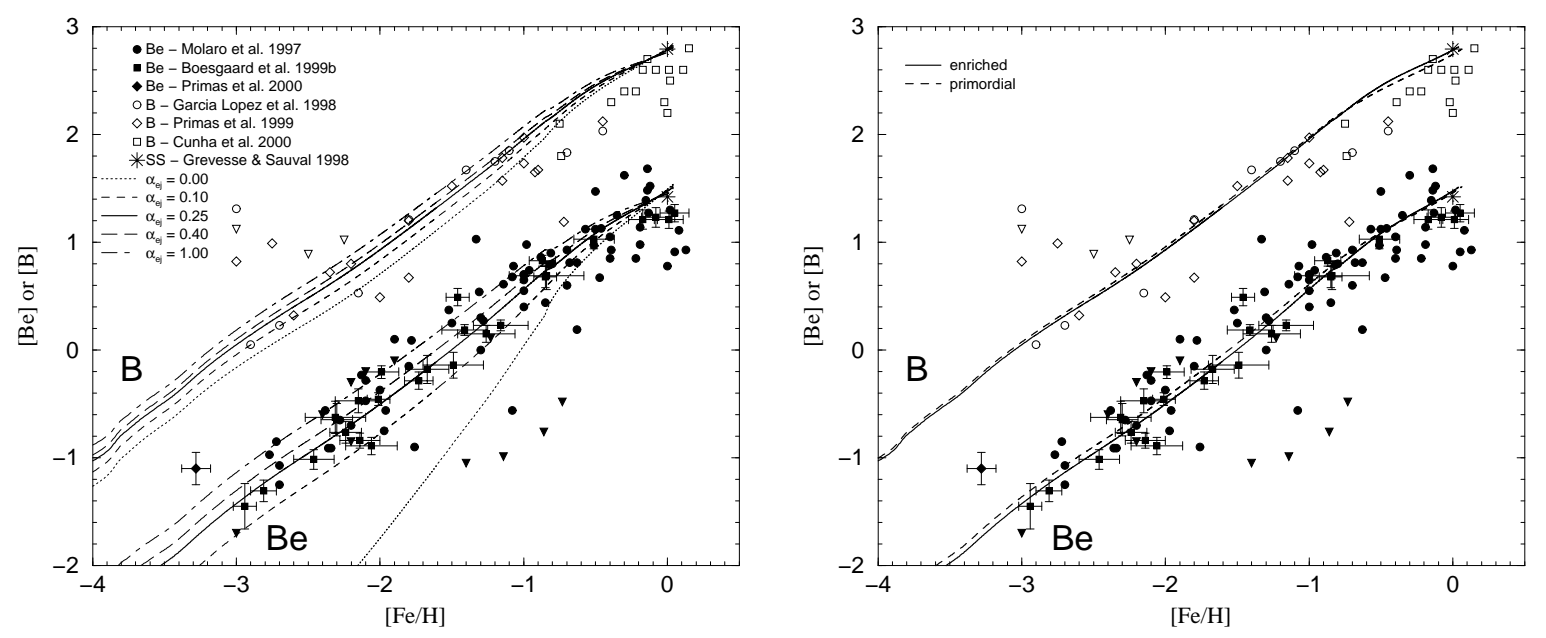

Fig. 3.- Beryllium and boron evolution. Left panel: evolution for the enriched model and different values of $\alpha_{\mathrm{ej}}$. Right panel: evolution for the $\alpha_{\mathrm{ej}}=0.25$ model and the two infall compositions. 


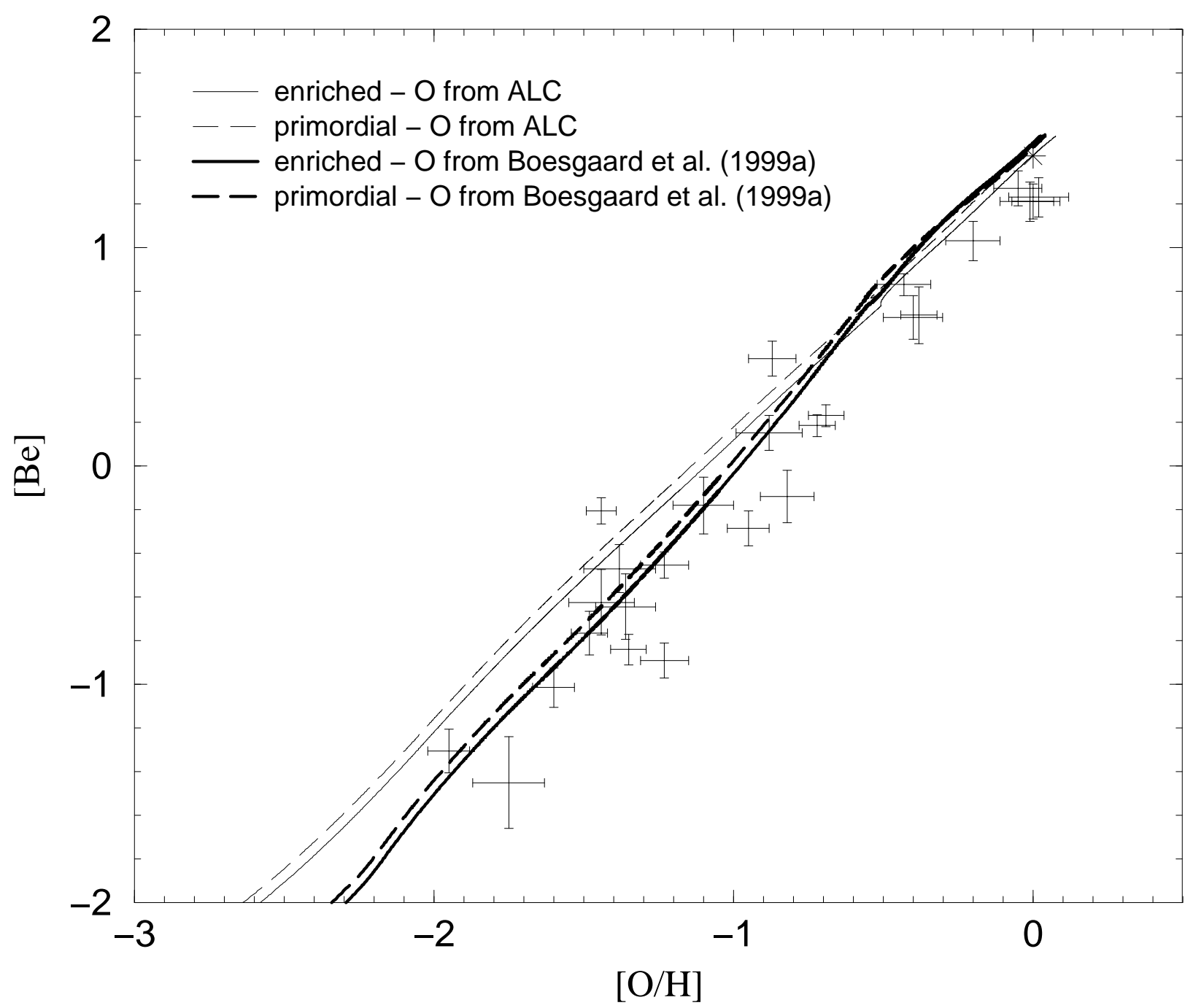

Fig. 4. - Beryllium vs oxygen evolution for the $\alpha_{\mathrm{ej}}=0.25$ case. Oxygen resulting from our model is used in thin line plots, while oxygen from Boesgaard et al. (1999a) is used in the thick line ones. Data: Boesgaard et al. (1999b). 

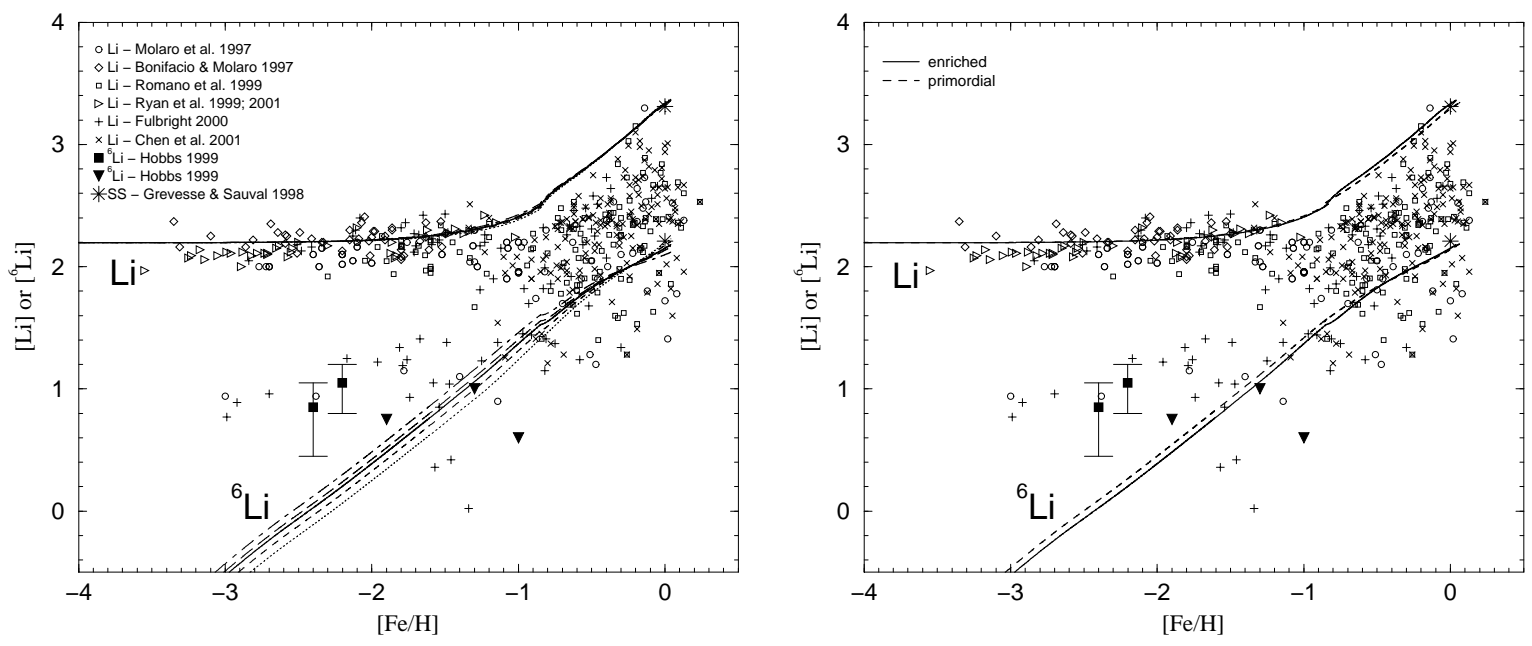

Fig. 5.- Lithium and ${ }^{6} \mathrm{Li}$ evolution. Left panel: enriched model and same values of $\alpha_{\text {ej }}$ as in figure 3. Right panel: both infall models for $\alpha_{\mathrm{ej}}=0.25$. 


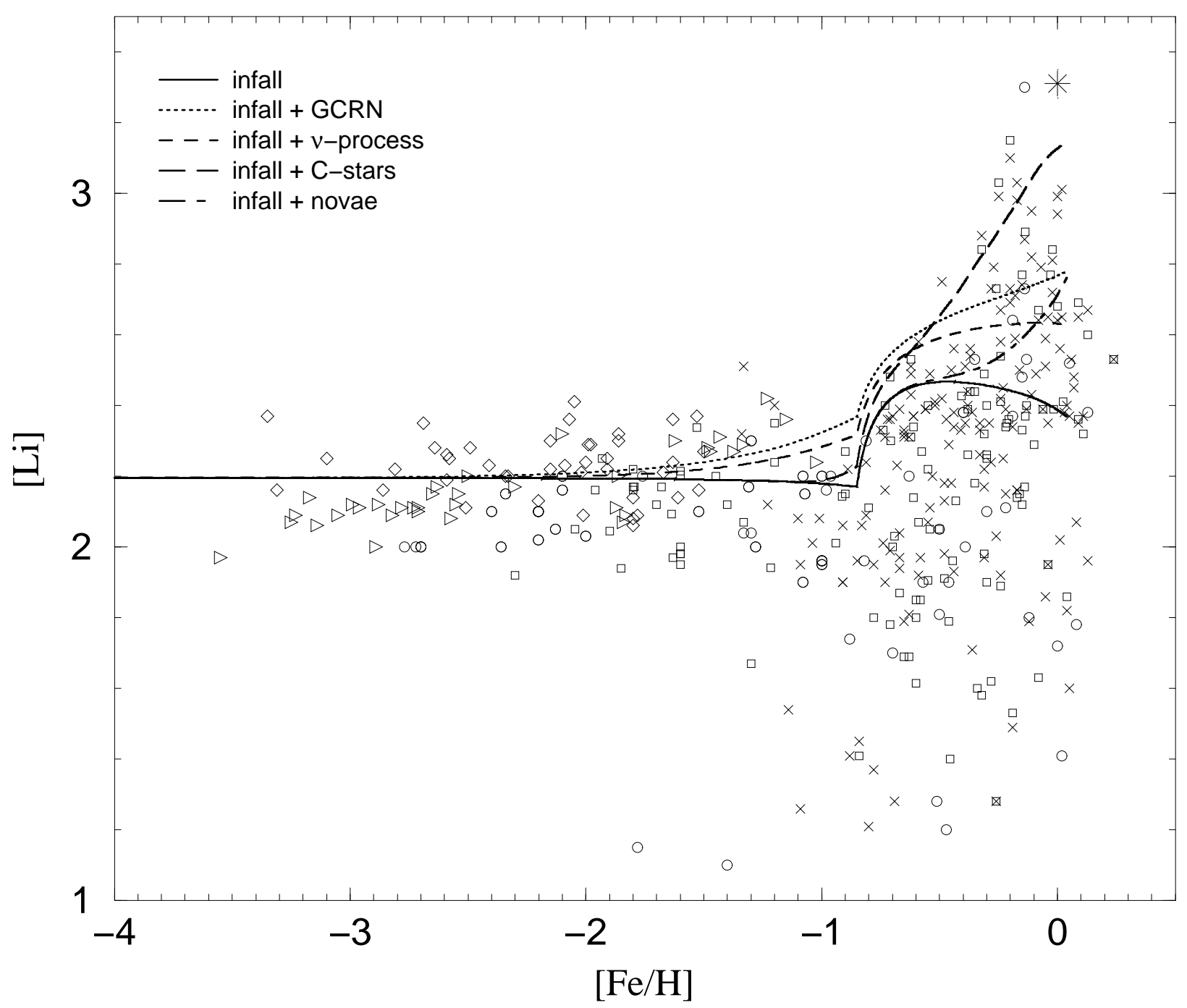

Fig. 6.-Different contributions for the lithium evolution, for the enriched infall composition and $\alpha_{\mathrm{ej}}=0.25$. 


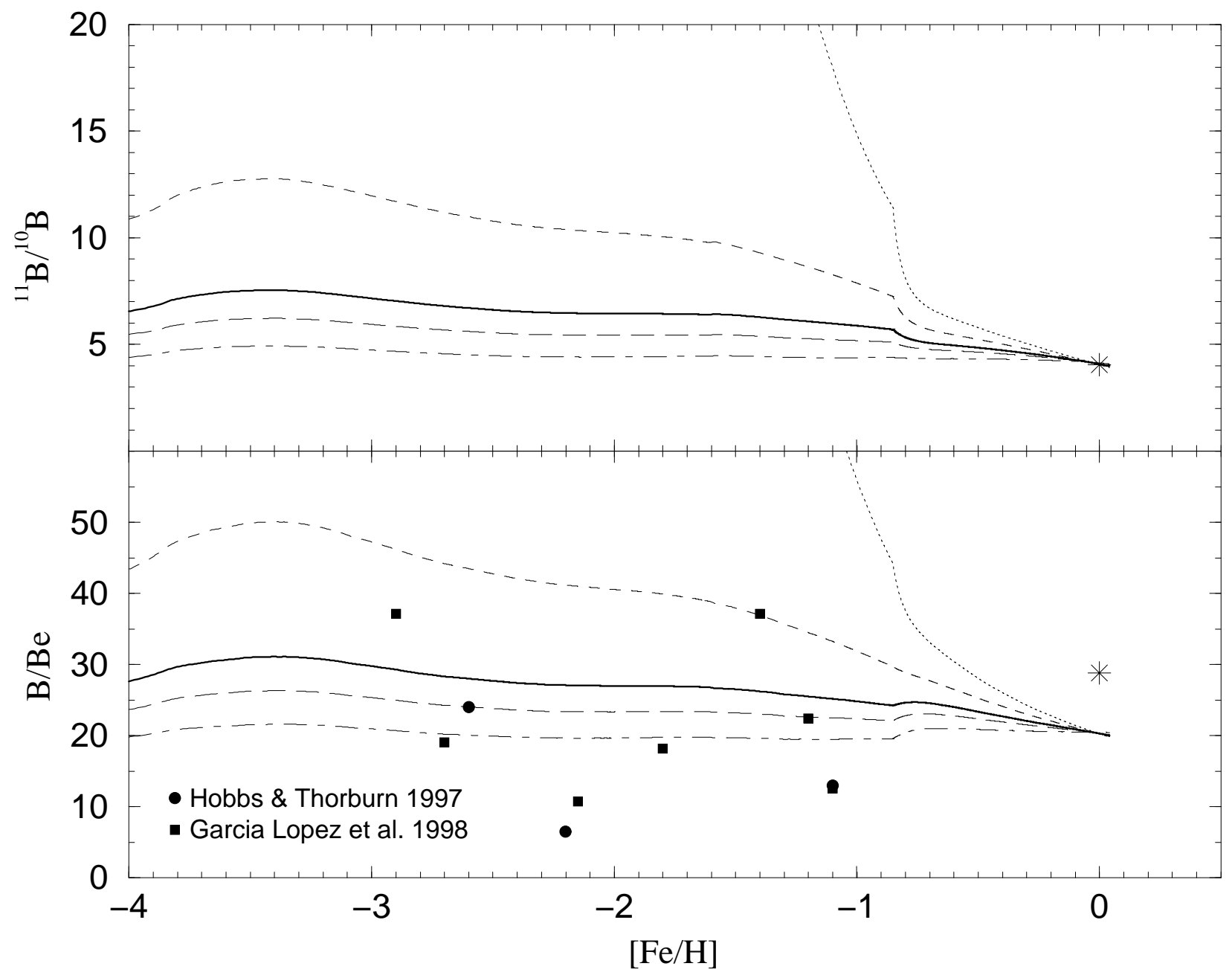

Fig. 7. $-{ }^{11} \mathrm{~B} /{ }^{10} \mathrm{~B}$ and $\mathrm{B} / \mathrm{Be}$ ratios evolution. Same values of $\alpha_{\mathrm{ej}}$ as in figure 3 , for the enriched infall composition. 


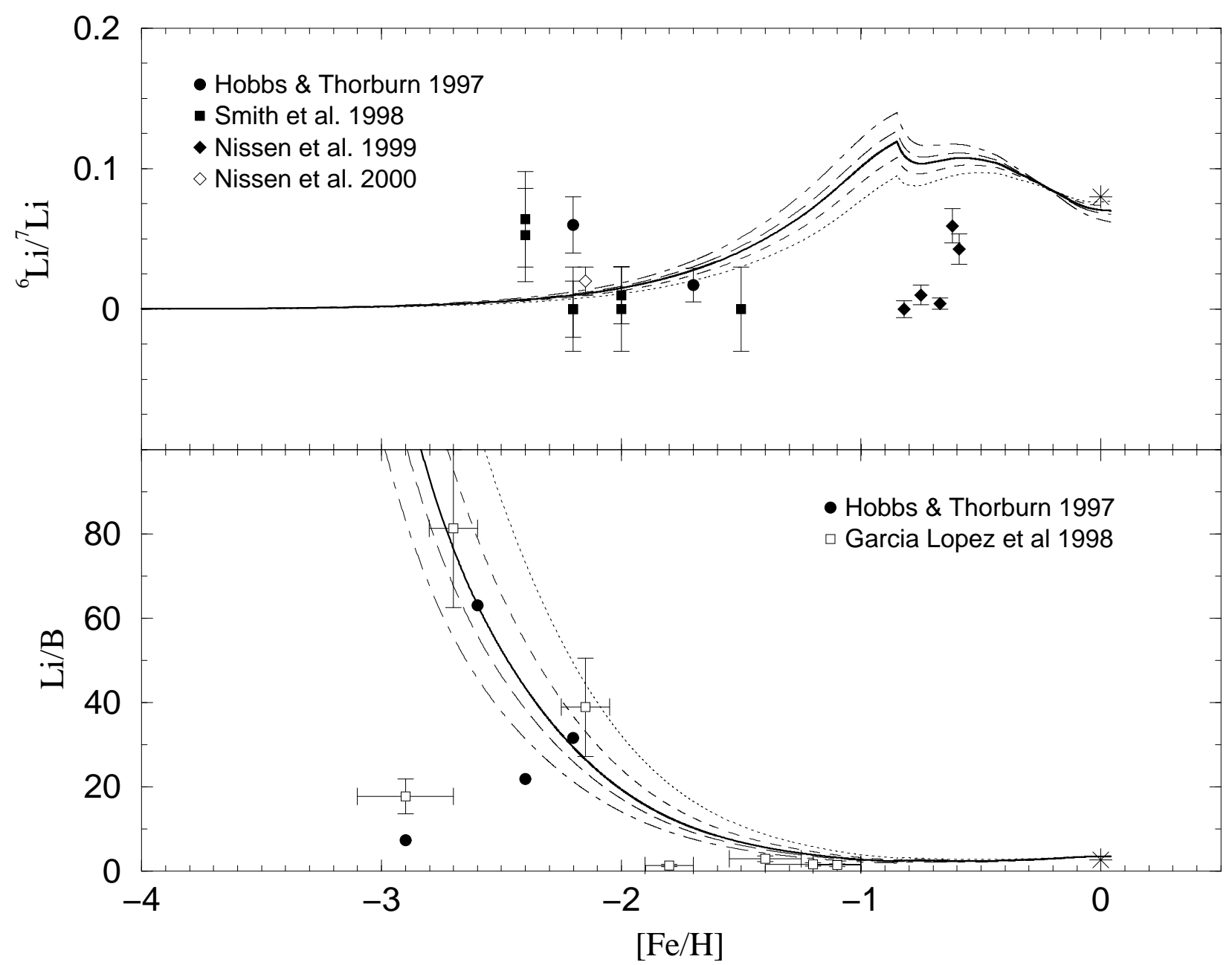

Fig. 8. $-{ }^{6} \mathrm{Li} /{ }^{7} \mathrm{Li}$ and $\mathrm{Li} / \mathrm{B}$ ratio evolution. Same values of $\alpha_{\mathrm{ej}}$ as in figure 3 , for the enriched infall composition. 


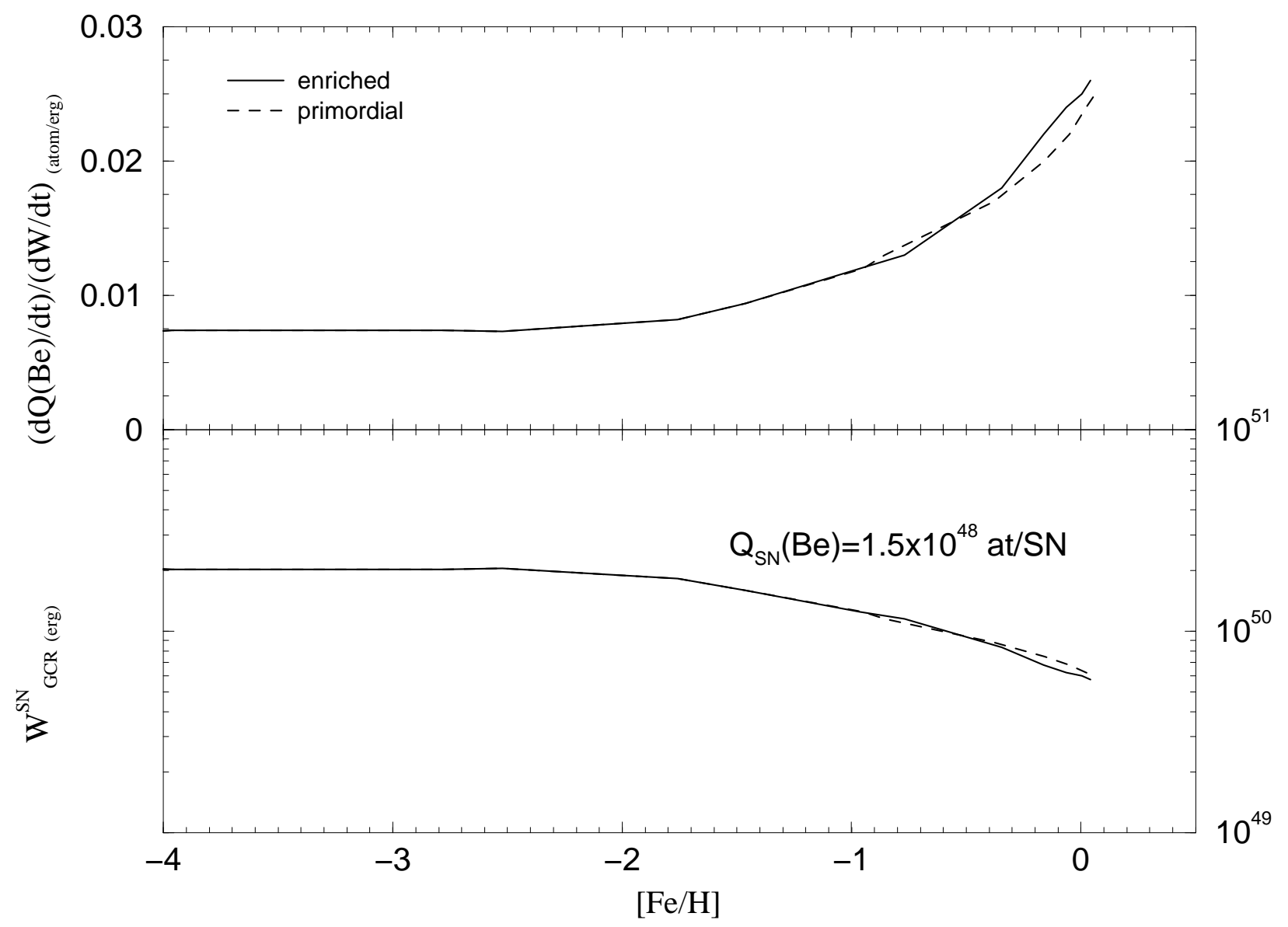

Fig. 9.- Upper panel: $Q(\mathrm{Be}) / \dot{W}$ vs $[\mathrm{Fe} / \mathrm{H}]$ in our $\alpha_{\mathrm{ej}}=0.25$ model. Lower panel: Energy per supernova needed to accelerate GCR, according to our evaluation of the atoms of beryllium produced by GCR for each SN event. 